\title{
Determinantes del crecimiento empresarial en el sector manufacturero colombiano
}

Manuela Carmona Gonzalez

Universidad del Quindío

Colombia

Yuliet Carvajal Muñoz Universidad del Quindío

Colombia

Saudy Milena Aguirre Serna

Universidad del Quindío

Colombia

Francisco José Ocampo Giraldo

Universidad del Quindío

Colombia

Adriana María Flórez Laiseca

Universidad del Quindío

Colombia 


\title{
Determinantes del crecimiento empresarial en el sector manufacturero colombiano
}

\author{
Manuela Carmona Gonzalez ${ }^{(1 D}$, Yuliet Carvajal Muñoz ${ }^{1}$, Saudy Milena Aguirre \\ Serna $^{1}$ (D) Francisco José Ocampo Giraldo ${ }^{1}$ y Adriana María Flórez Laiseca ${ }^{1 D}$ \\ 1 Universidad del Quindío, Colombia
}

Para citaciones: Carmona, M., Carvajal, Y., Aguirre, S., Ocampo, F., Flórez, A. (2020). Determinantes del crecimiento empresarial en el sector manufacturero colombiano. Panorama Económico, 28(1), 1-15. https://doi.org/10.32997/pe-2020-2665

Recibido: 21/08/2019

Aprobado: 19/11/2019

Autor de correspondencia:

Manuela Carmona Gonzalez

mcarmonag_1@uqvirtual.edu.co

Editor: Andrés Escobar Espinoza Universidad de Cartagena, Colombia.

Tipología IBN Publindex: Artículo de investigación científica y tecnológica.
Copyright: (c 2020. Carmona, M., Carvajal, Y., Aguirre, S., Ocampo, F., Flórez, A. Este es un artículo de acceso abierto, distribuido bajo los términos de la licencia https://creativecommons.org/licenses/bync-sa/4.0/ la cual permite el uso sin restricciones, distribución y reproducción en cualquier medio, siempre y cuando que el original, el autor y la fuente sean acreditados.

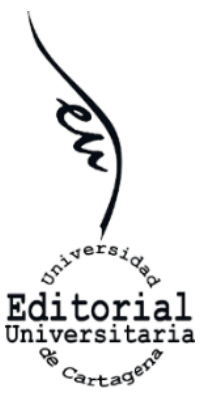

\section{RESUMEN}

El presente documento analiza los determinantes del crecimiento empresarial del sector manufacturero en Colombia, con particular énfasis en factores internos como el capital humano, el valor agregado, el valor de las ventas, los costos de producción, administración y ventas, con base la teoría del crecimiento proporcional de Gibrat (1931). Se tuvo en consideración las diferencias entre departamentos y se realizó una estimación econométrica de datos panel para el periodo 2008-2016, empleando para tal fin la información estadística de la Encuesta Anual Manufacturera del DANE. Las variables que influyen en el crecimiento empresarial de las empresas del sector manufacturero son: costo de producción, administración y ventas, valor agregado y años de escolaridad. Si se considera la aglomeración de departamentos según niveles del PIB, aquellos con niveles de ingresos bajos y medios, carecen de mano de obra calificada. Lo contrario sucede con departamentos que presentan niveles de ingresos altos quienes se convierten en receptores de la mano de obra calificada, lo que explica el incremento en las ventas y el valor agregado en los departamentos.

Palabras clave: Crecimiento empresarial; Industria; Panel de datos. JEL: D21, D24

\section{Firms' growth determinants in the colombian manufacturing sector}

\begin{abstract}
This document analyzes -based on the Gibrat's theory on proportional growth- the determining factors of business growth in the manufacturing sector in Colombia, with particular emphasis on internal factors such as human capital, added value, value of sales, costs of production, administration and sales. Differences between departments were taken into account and an econometric estimation of panel data was made for the period 2008-2016, using for this purpose the statistical information from DANE's Annual Manufacturing Survey. The variables that influence the business growth of companies in the manufacturing sector are: cost of production, administration and sales, added value and schooling years. Considering the agglomeration of departments according to GDP levels, those with low and middle income levels lack qualified labor. The opposite is the case with departments with high income levels that become hosts of skilled labor, which explains the increase in sales and added value in the departments.
\end{abstract}

Keywords: firm's growth; manufacturing sector; Panel data.

JEL: D21, D24 


\section{INTRODUCCIÓN}

En los últimos 20 años, las economías de América Latina y el Caribe han venido experimentado un crecimiento económico sostenido y una expansión regional que se acerca al 3\% anual, tal y como lo reporta la Organización para la Cooperación y el Desarrollo Económico (OCDE, 2016: 4). Este comportamiento podría reflejarse entre muchas variables, por el fenómeno de desarrollo de las empresas. Por ello, con el fin de comprender el desarrollo empresarial se requiere inicialmente identificar los determinantes del crecimiento. Al respecto, varios autores como Gibrat (1931), Penrose (1959) y Perroux (1963) coinciden en el análisis de factores internos tales como el capital humano, el valor agregado, el valor de las ventas, los costos de producción administración y las ventas entre otros, sumados a elementos externos como las políticas macroeconómicas que también generan un impacto directo. Por ende, el presente artículo se enfoca en el análisis de los factores internos que determinan el crecimiento económico de las empresas especialmente las del sector manufacturero.

Por otro lado, las disparidades en el desempeño económico intrarregional que se observan a partir de los múltiples estudios empíricos para Colombia (Cuervo, 2003; Zuleta, 2007; Banco de la Republica, 2007; López y Sánchez, 2008 y Rodríguez, 2014) y la connotación de las empresas como motor del desarrollo económico, justifican nuevas explicaciones en torno a los determinantes profundos del crecimiento empresarial. De esta manera, se evalúa de cerca el crecimiento empresarial y la causalidad entre el ingreso per cápita y la dinámica empresarial, lo que permite introducir dos novedades en relación con investigaciones afines (Trujillo y Iglesias, 2012; Moreno y Ramos, 2013), la primera enfocada en la agrupación de los departamentos de acuerdo al nivel de ingreso, con el fin de identificar el impacto que generan las variables de estudio en cada grupo de departamentos, y la segunda con la implementación de técnicas de econometría para datos panel con el objetivo de capturar los efectos fijos regionales.

Ahora bien, teniendo en cuenta la información del Departamento Administrativo Nacional de Estadística (DANE), para 21 de los 32 departamentos de Colombia, se identificaron las variables internas de las empresas manufactureras que inciden en su crecimiento económico. Adicionalmente, se utilizó una metodología descriptiva mediante mapas de calor, donde se ubica geográficamente los departamentos con mayor crecimiento empresarial a lo largo del tiempo y una metodología econométrica de datos panel, donde se efectúa una aglomeración por nivel bajo, medio y alto en relación con el tamaño del Producto Interno Bruto (PIB) manufacturero, para los departamentos; siendo este un elemento innovador. A continuación, se aborda el marco teórico y antecedentes que contextualizan el estudio, seguido de la metodología y el planteamiento del modelo econométrico utilizado para la validación y análisis de los determinantes del crecimiento empresarial, para finalizar con los resultados y las conclusiones.

\section{REVISIÓN DE LITERATURA}

Uno de los autores que aborda principalmente el crecimiento empresarial es Gibrat (1931), citado por Blázquez, Dorta, y Verona (2006), el cual estableció la hipótesis más reconocida en el estudio del crecimiento de la empresa, denominada la ley del efecto proporcional. En ella se describe el crecimiento como el resultado de diversas fuerzas o factores que 
actúan de forma proporcional en el tamaño de las empresas; ya que según Blázquez et al. (2006): "el crecimiento de las empresas es un proceso estocástico originado por la acción de innumerables e insignificantes factores aleatorios que actúan de forma proporcional sobre el tamaño de las empresas" (p.44).

En efecto, existen factores que se desarrollan al interior de las empresas, los cuales pueden ser controlados por los agentes administrativos involucrados, ya que esta teoría sostiene que el crecimiento se genera gracias a las fuerzas decisivas que se encuentran al interior del lugar. De esta manera, se estudian elementos como el capital humano, la innovación y el conocimiento que, sumados a la teoría del crecimiento y desarrollo endógeno (Vázquez, 2018), constituyen un proceso de crecimiento económico y de cambio estructural, que conduce al mejoramiento en la calidad de vida de la población. Puesto que como menciona Romero (2002), la dimensión económica se caracteriza "por ser un sistema específico de producción que permite a los empresarios locales usar eficientemente los factores productivos y alcanzar niveles de productividad suficientes para ser competitivos en los mercados" (p. 144).

Una vez identificados los factores internos de la empresa como pilar fundamental del crecimiento, se busca canalizar el enfoque en el capital humano, debido a que como lo sustentan London y Formichella (2006) "pone mayor énfasis en el rol del ser humano como productor de bienes y servicios, por lo cual se observa y se estudia cómo cada día se mejora en la calificación del hombre para que éste sea más productivo" (p.20), de lo que se desprende, la significancia de éste como impulsor de la economía. Del mismo modo, Pérez y Castillo (2016) señalan que dichas obras del capital humano aportaron a la teoría económica para que se basaran principalmente en los años de formación del personal y en la experiencia profesional, ya que estas explican el incremento en el producto y en su valor agregado.

Teniendo en cuenta que el crecimiento empresarial es uno de los temas con mayor relevancia política y privada, se puede traer a colación lo que Daza (2015) propone en su investigación, en la cual define el crecimiento empresarial como un proceso aleatorio independiente del tamaño de la empresa, y que, a su vez, existen influencias de factores internos y externos en el crecimiento de la organización. En este sentido, una de las variables propuestas reagrupa los costos de producción, la administración y las ventas, tal y como lo expone Andrade, Olivares y Robles (2014), puesto que mientras más conocimiento se tenga sobre estos temas, más planificación consciente se deriva en el proceso productivo y administrativo y, por ende, se logrará un control eficaz en estas áreas.

Del mismo modo, Olivares y Robles (2014) destacan que:

Para lograr una mayor eficiencia en la planeación y el control de los procesos de producción y administración, es necesario medir y revisar las diferentes alternativas de planeación, financiera, económica y de toma de decisiones para simplificar los sistemas de producción. (p. 80)

En otras palabras, mientras haya mayor conocimiento de los costos, habrá mayor eficiencia, lo cual conlleva a que los empresarios obtengan mejores resultados y así 
aportar a un crecimiento empresarial. También, se identifica que un factor determinante para el crecimiento de las Pymes es la innovación; afirmando que los factores financieros y humanos, tienen elementos fundamentales para alcanzarla, ya que el no generar conocimiento y aprendizaje en el personal limita su crecimiento. Igualmente, para fomentar el crecimiento y la competitividad de las pymes, según Rocca, García y Duréndez (2016) es fundamental tener prácticas eficientes en cuanto a la gestión de los recursos humanos, obteniendo así una mayor probabilidad de éxito en el mercado.

Finalmente, es necesario reconocer la relevancia que presenta el capital humano en el impacto del crecimiento empresarial y que este ha despertado gran interés al interior de las firmas. Se destaca el interés de Cañibano (2011) cuando afirma que "las probabilidades de que los conocimientos de un individuo sean más productivos aumentan cuando éste se desenvuelve en un entorno en el que hay un alto nivel general de capital humano" ( $p$. 258). Por lo anterior, se puede decir que se tendría un efecto productivo, si se aumenta el desarrollo de capital humano y se aporta al entorno.

\section{METODOLOGIA}

Para evaluar los determinantes del crecimiento de las empresas industriales en Colombia, se parte de la aplicación de un método descriptivo y cuantitativo, utilizando para estos los softwares GeoDa y STATA respectivamente. Adicional, se hace uso de información secundaria suministrada por el DANE (2016) y reportada en la Encuesta Anual Manufacturera (EAM), que abarca empresas que contratan de 1-50 empleados y a su vez, reportan ingresos moderados inferiores a los 5.000 SMLMV (Pymes) (Grupo Bancolombia, 2018). A partir de ello, se construye una base consolidada por microdatos correspondiente al periodo comprendido entre 2008 y 2016. Para este estudio se excluyen los departamentos de Caquetá, Chocó, La Guajira, Arauca, Putumayo, San Andrés y Providencia, Casanare, Vichada, Vaupés, Guainía, Guaviare y Amazonas, debido a la ausencia de información.

Ahora bien, para el procesamiento y tratamiento de la información se hace uso de un modelo econométrico para datos panel con efectos fijos, es decir, aquellos que responden al conjunto de observaciones que pueden ser analizadas temporalmente y transversalmente, estimando así el grado de integración existente entre las variables de estudio. En cuanto a los efectos fijos, estos permiten evaluar los impactos de cada departamento a nivel individual. Adicional, en el análisis descriptivo se implementan mapas de calor donde se identifican los departamentos según el nivel de ingresos como lo muestra la siguiente tabla:

Tabla 1. Departamentos según ingreso PIB manufacturero.

\begin{tabular}{clll}
\hline Ingresos bajos & \multicolumn{2}{l}{ Ingresos medios } & \multicolumn{2}{l}{ Ingresos altos } \\
\hline 1. Magdalena & 1. Norte de Santander & 1. Atlántico \\
2. Cesar & 2. Boyacá & 2. Bolívar \\
3. Sucre & 3. Caldas & 3. Santander \\
4. Córdoba & 4. Risaralda & 4. Antioquia \\
5. Quindío & 5. Tolima & 5. Cundinamarca \\
6. Huila & 6. Cauca & 6. Bogotá D.C \\
7. Nariño & 7. Meta & 7. Valle del Cauca \\
\hline
\end{tabular}

Fuente: elaboración propia con base en datos del DANE. 
Esta metodología ha sido implementada para diferentes estudios del crecimiento empresarial, para el caso Colombiano Trujillo y Iglesias (2012), realizan un estudio para las micro, pequeñas y medianas empresas en el sector metalmecánico, estos estiman tres paneles dinámicos para analizar el crecimiento de las mypymes, los resultados arrojan que la competencia local y los costos laborales, explican el crecimiento empresarial. Para el caso de Latino América, Valdez y Noda (2018), aplican datos panel para encontrar los factores que determinan el aumento o disminución de las empresas en México, donde utilizan la variable edad de la empresa, PIB, PIB per cápita, entre otras.

Al igual que Gómez, Mena y Beltrán (2018), adicional en Perú, hacen uso de datos panel para determinar los factores que influyen en el crecimiento de las cajas municipales, donde utilizan variables de tipo financiero y en España, Guisado, Coca, y Guisado (2016), aplican un panel de innovación para analizar la influencia del tamaño en empresarial y la actividad exportadora sobre la productividad laboral de las empresas y donde se enfocan principalmente en las empresas manufactureras españolas. Seguidamente en la tabla 2 se identifican y se caracterizan las variables que impactan en el crecimiento empresarial

Tabla 2. Variables de estudio

\begin{tabular}{|c|c|c|}
\hline Variables & Subvariables & Fuente \\
\hline \multirow[t]{3}{*}{ Ingreso } & - Valor agregado & $\begin{array}{l}\text { Valor agregado: es el total de los ingresos recibidos por } \\
\text { el uso de los factores productivos participantes en el } \\
\text { proceso de producción. Se calcula como la diferencia } \\
\text { entre el valor de la producción bruta y los consumos } \\
\text { intermedios empleados. }\end{array}$ \\
\hline & $\begin{array}{l}\text { - Costo de producción, } \\
\text { administración y } \\
\text { ventas }\end{array}$ & $\begin{array}{l}\text { Costos de producción, administración y ventas: gasto } \\
\text { anual por concepto de la producción administración y } \\
\text { ventas de la empresa. }\end{array}$ \\
\hline & - Valor de las ventas & $\begin{array}{l}\text { Valor de las ventas: es el valor de ventas reportadas por } \\
\text { las empresas manufactureras en cada año. }\end{array}$ \\
\hline Capital humano & $\begin{array}{l}\text { Nivel de formación } \\
\text { del personal }\end{array}$ & $\begin{array}{l}\text { Total, de años académicos cursados por las personas de } \\
\text { cada departamento }\end{array}$ \\
\hline Institucionalidad & Número de empresas & Total, de observaciones tomadas por la EAM \\
\hline
\end{tabular}

Se evalúa estas variables teniendo en cuenta la teoría económica y administrativa sobre el crecimiento empresarial, las cuales reflejan los factores internos del crecimiento económico de las empresas. Destacando el estudio realizado por el Banco de la Republica (2008) sobre los determinantes del crecimiento de las empresas no financieras en Colombia, realizaron una revisión de literatura donde destacan tres resultados importantes, la primera es donde refutan la teoría de Gibrat ya que las empresas grandes presentan tasas menores de crecimiento en comparación con las empresas pequeñas, la relación entre el tamaño de las empresas y su edad es negativa evidenciando que para las empresas más pequeñas una mayor edad significa mayor crecimiento, en tanto que para las más grandes no ocurre lo mismo.

Por otro lado, el estudio realizado por el Consejo Económico y Social de Aragón (2018), utilizan la teoría del Gibrat para evaluar el crecimiento y la rentabilidad empresarial en Aragón, donde también caracterizan y estratifican las rentabilidades como alta, media y baja para evaluar su crecimiento y evolución. A su vez Morales; Valle; Freire Torres, y Silva (2017), evalúan el crecimiento del sector de las carrocerías en Ecuador, en el cual 
identifican los factores internos financieros de las empresas, donde utilizan las variables ventas, utilidad y capacidad instalada.

\section{Modelo econométrico}

Es importante resaltar que, la variable dependiente hace referencia a las ventas generadas por las empresas manufactureras en un periodo determinado. Por otro lado, las variables explicativas corresponden a Valor Agregado, Costo de producción, administración y ventas y Nivel de formación del personal. Vale aclarar que se aplicó logaritmo a todas las variables para suavizar la serie y no tener problemas de escala. Este modelo se plantea de la siguiente forma:

Regresión lineal datos panel con efectos fijos

$$
\log \left(Y_{i t}\right)=\alpha_{i}+\beta_{1} \log \left(x_{1}\right)_{i t}+\beta_{2} \log \left(x_{2}\right)_{i t}+\beta_{3} \log \left(x_{3}\right)_{i t}+\varepsilon_{i t}
$$

Remplazando:

$\log \left(\right.$ Ventas $\left._{i t}\right)=\alpha_{i}+\beta_{1} \log (\text { Valoragregado })_{i t}+$

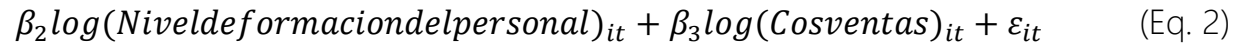

Donde la variable dependiente Yit hace referencia a las ventas manufactureras del departamento $i$ en el periodo $t$, las cuales según la hipótesis planteada tendrían que verse afectadas de una forma muy significativa frente a los cambios de las variables explicativas, las cuales son, Valor agregado, Nivel de formación del personal y Costo de producción, administración y ventas, por último el efecto individual está representado por $\alpha_{i}$ y los

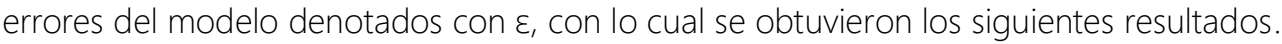

\section{RESULTADOS}

Los resultados están divididos en dos secciones, la primera hace referencia a los resultados descriptivos a través de los mapas de calor y la segunda son los resultados econométricos arrojados por el modelo panel implementado. En la figura 1 se muestra el PIB manufacturero en miles de millones para cada departamento en diferentes años según los datos del DANE. A partir de la figura 1, podemos identificar que los departamentos con mayor aporte en cuanto al PIB manufacturero a lo largo del tiempo son Antioquia, Valle del Cauca, Santander, Cundinamarca, Atlántico y Bogotá D.C. Esto es explicado por la competitividad empresarial; la cual hace referencia a la capacidad de un negocio para producir y vender productos y servicios que cumplan con la calidad de los mercados, al mismo precio o con precios más bajos, y maximizando los rendimientos de los recursos consumidos para producirlos. Y estos departamentos según el consejo privado de competitividad se encuentran entre los 7 departamentos más competitivos a nivel nacional. 
Figura 1. PIB manufacturero por departamentos

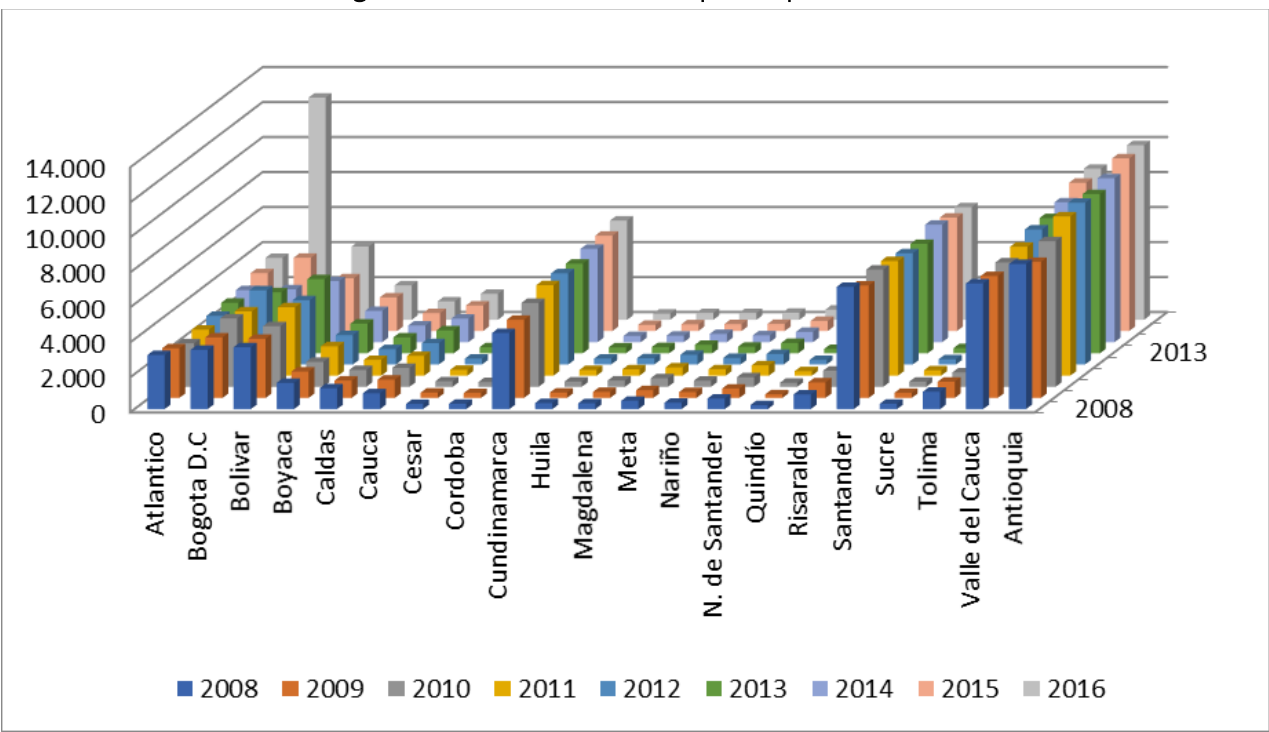

Fuente: Elaboración propia.

A continuación, se muestran los mapas de calor, donde se identifican los departamentos de ingresos alto, medio y bajo según su PIB manufacturero esto con el fin de que el lector se ubique geográficamente, adicionalmente la leyenda de estos mapas indica que el color más claro se asocia con los departamentos de ingresos más bajos, el intermedio con ingresos medios y el oscuro con ingresos altos y finalmente el color negro indica que no poseen información.

En la figura 2 se observa la estratificación de los departamentos según el ingreso del PIB manufacturero. Además, en los mapas se pueden evidenciar el código que identifica cada departamento según el DIVIPOLA del DANE; tal como se muestra a continuación:

Tabla 3. Codificación de los departamentos

\begin{tabular}{cccccc}
\hline COD & Departamento & COD & Departamento & COD & Departamento \\
\hline 05 & Antioquia & 27 & Chocó & 73 & Tolima \\
08 & Atlántico & 41 & Huila & 76 & Valle del \\
& Cauca \\
11 & Bogotá D.C & 44 & La Guajira & 81 & Arauca \\
13 & Bolívar & 47 & Magdalena & 85 & Casanare \\
15 & Boyacá & 50 & Meta & 86 & Putumayo \\
17 & Caldas & 52 & Nariño & 91 & Amazonas \\
18 & Caquetá & 54 & Norte de & 94 & Guainía \\
& & & Santander & & \\
19 & Cauca & 63 & Quindío & 95 & Guaviare \\
20 & Cesar & 66 & Risaralda & 97 & Vaupés \\
23 & Córdoba & 68 & Santander & 99 & Vichada \\
25 & Cundinamarca & 70 & Sucre & & \\
\hline
\end{tabular}

Fuente: Elaboración propia

Los departamentos con ingreso alto son: Valle del Cauca, Bogotá D.C, Cundinamarca, Antioquia, Santander, Bolívar y Atlántico. Los departamentos con ingreso medio son: 
Cauca, Tolima, Risaralda, Caldas, Boyacá y Norte de Santander. Los departamentos con ingreso bajo son: Nariño, Huila, Quindío, Córdoba, Sucre, Magdalena y Cesar. En la figura 3 se observa el nivel de formación del personal por departamento, haciendo una comparación entre el año 2008 y 2016, con el fin de observar los cambios en los diferentes departamentos y en cuales esta variable incide de una manera significativa.

\section{Figura 2. Departamentos de Colombia por nivel de ingreso según PIB manufacturero}

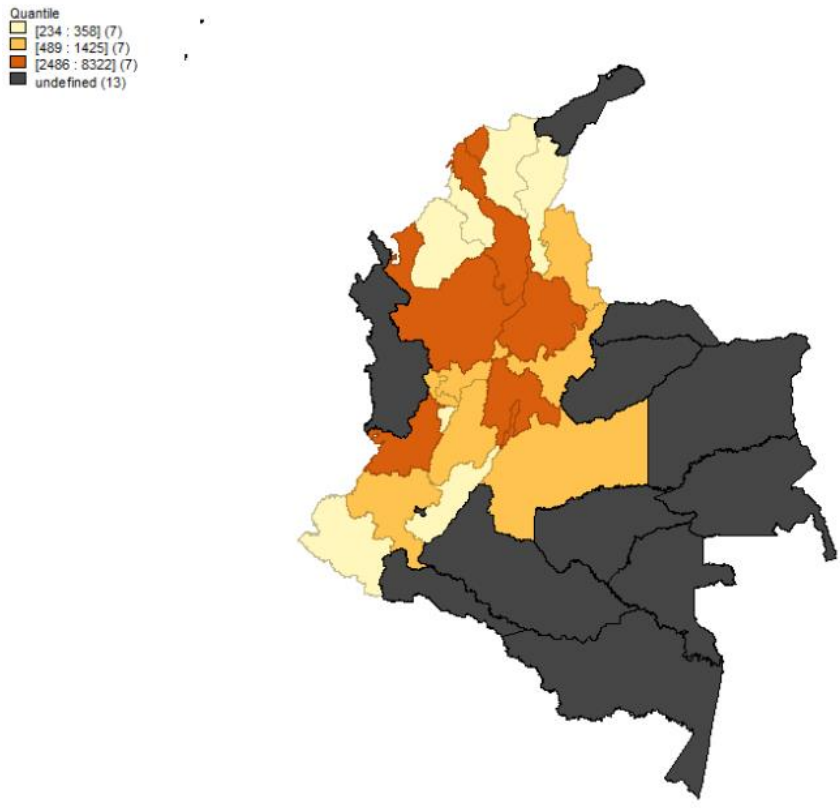

Fuente: Elaboración propia.

Figura 3. Nivel de escolaridad por departamentos
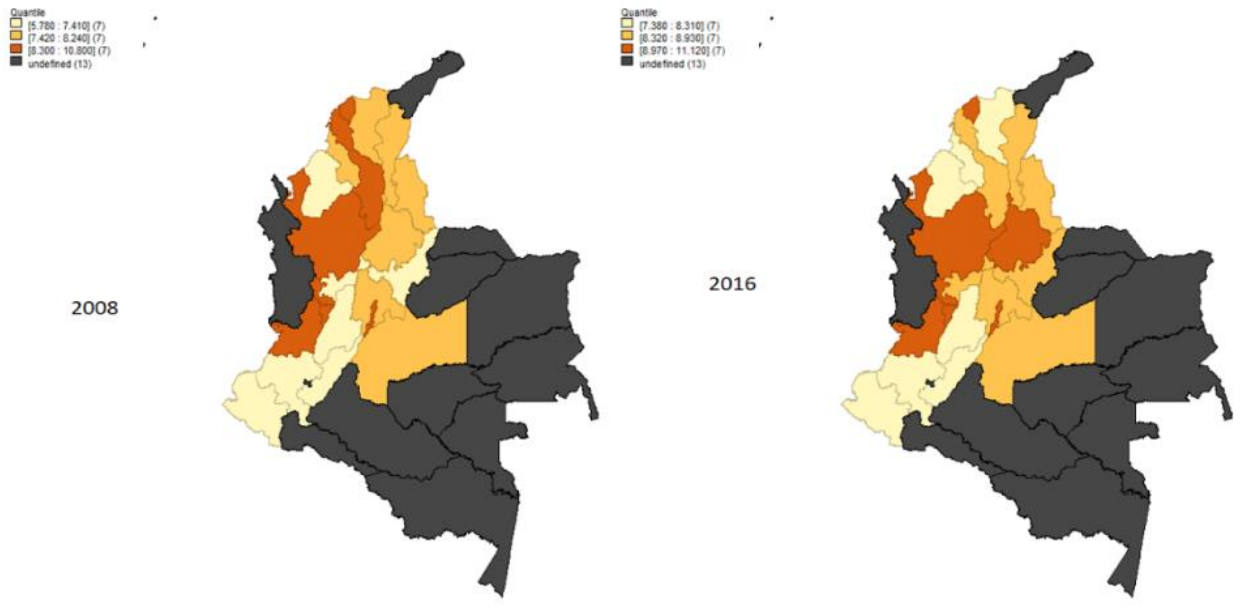

Fuente: Elaboración propia.

Como se puede evidenciar en el mapa anterior, los departamentos del Valle del Cauca, Antioquia y el Distrito Capital se mantienen en el tiempo con mayores niveles de formación del personal, esto es debido a que son focos de concentración de mano de obra calificada, explicado por factores como los salarios, los cuales en cierta medida 
incrementan el nivel de productividad que han alcanzado estos departamentos hegemónicos. Esto se conoce como fuga interregional de cerebros, que se refiere a la migración de personas altamente educadas hacia regiones más prósperas, ya que en muchos casos es la alternativa más favorable para aumentar la calidad de vida de una persona y más partiendo de un país como Colombia, donde las diferencias regionales tanto en términos del PIB, como en las condiciones del mercado laboral, son grandes (Cepeda Emiliani, S.F).

Respecto a la Costa Atlántica se demuestra una pérdida de forma generalizada en el capital humano (nivel de formación del personal), como resultado de una disminución en el tiempo para los departamentos de Sucre, Bolívar y Magdalena. Este decrecimiento se debe en gran proporción a las condiciones laborales inestables en comparación con otros departamentos, adicional, el Banco de la República en su estudio Evolución socioeconómica de la región Caribe colombiana entre 1997 y 2017 encontraron como principales problemas de la región la baja dotación del capital humano y la ineficiencia e ineficacia del sector público regional y la cobertura en educación básica registró un lento crecimiento a lo largo del tiempo (Banco de la República, 2017).

Lo cual involucra fuertemente las políticas públicas de cada departamento y lleva a reflexionar que incentivos deben ofrecer las regiones para atraer a las personas más calificadas y retener a su propio capital humano, evitando así las fugas de capital hacia otras regiones. Finalmente, un fenómeno opuesto se presentó en departamentos más centrales como Boyacá y Caldas, los cuales se vieron fortalecidos con el tiempo. Por otro lado, en la figura 4 se muestra el número de empresas para cada departamento en los años de estudio, esto con el fin de observar su evolución.

Figura 4. Número de empresas manufactureras por departamento

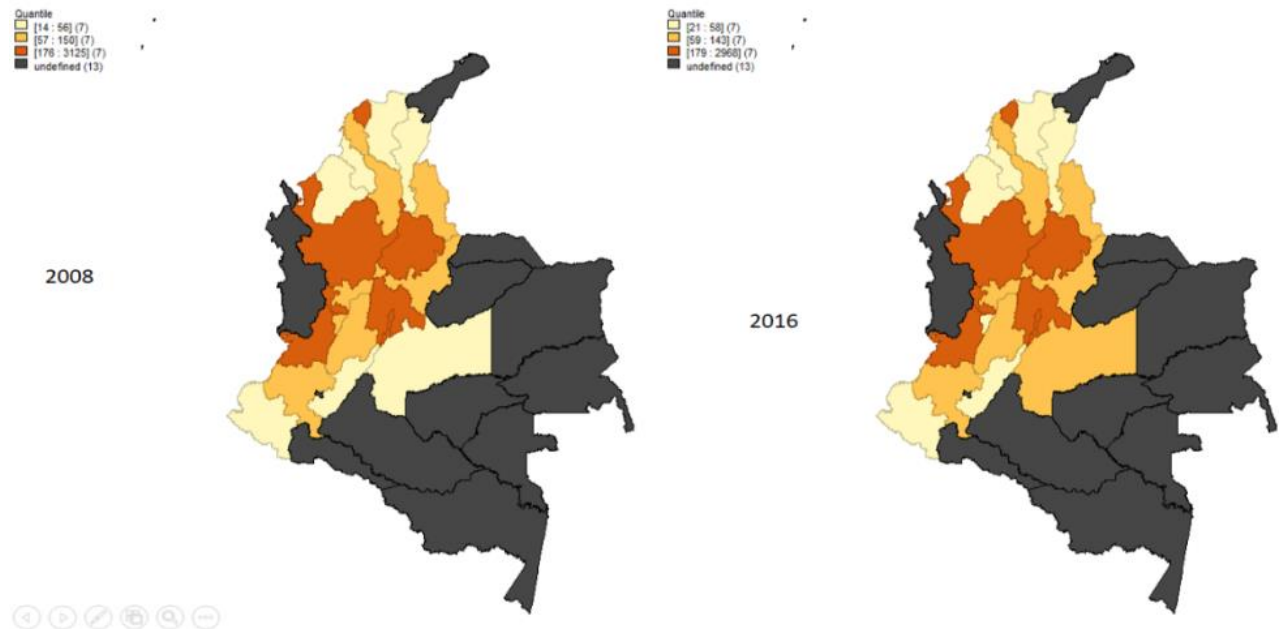

Fuente: Elaboración propia.

Teniendo en cuenta la figura 4., se puede evidenciar que los departamentos con ingresos altos, son los que presentan mayor número de empresas a lo largo del tiempo, estos departamentos presentan las condiciones más adecuadas para generar un desarrollo 
empresarial, debido a que son focos atractivos, tanto para inversionistas como para la mano de obra calificada; ya que la (OCDE, 2008) establece que "la inversión extrajera directa es una fuente importante de recursos para las economías locales, mejorando su competitividad, a través, principalmente de la transferencia tecnológica y del intercambio de experiencias entre la economía receptora y la inversora". Las empresas, por lo general buscan punto central para posicionarse en un país y así tener acceso a nuevos mercados, tanto nacionales como internacionales. (Fuentes Lopez, Jimenez Reyes, \& Perez Forero, 2019).

Adicionalmente se puede observar que se generan variaciones, en algunos departamentos respecto al número de empresas, entre ellos el Quindío que se ha visto disminuido en su densidad empresarial, debido a múltiples factores internos que les han quitado competitividad en el mercado laboral, así mismo, según el informe de coyuntura económica regional la industria manufacturera completo 4 años de débil desarrollo ya que las confecciones registraron descenso tanto en la producción como en las ventas y las exportaciones no tradicionales del Quindío según las clasificación internacional industrial uniforme, se evidencio un decrecimiento en un 60\% en el año 2015 en comparación con el año anterior (ICER, 2016).

\section{Modelo Panel}

A continuación, se muestra los resultados econométricos de datos panel para cada uno de los grupos de departamentos estratificados, esto permite un resultado diferenciador para evaluar los efectos en cada uno de ellos.

Tabla 4. Resultados de las estimaciones

\begin{tabular}{llll}
\hline & Menores ingresos & Ingresos medios & Ingresos altos \\
\hline Ecuación & & & \\
Lncosventas & $0.203^{\star \star \star}(4.91)$ & $0.113^{\star}(2.55)$ & $0.227(0.56)$ \\
Lnaosescolaridad & $0.092(0.23)$ & $0.346^{\star}(1.31)$ & $2.035^{\star \star *}(4.87)$ \\
Lnvaloragregado & $0.391^{\star \star \star}(6.35)$ & $0.789^{\star \star \star}(13.03)$ & $1.024^{\star \star *}(25.52)$ \\
Constante & $7.21^{\star \star *}(6.13)$ & $1.804^{\star \star *}(2.13)$ & $-4.364^{\star \star *}(-4.70)$ \\
No. de observaciones & 62 & 62 & 63 \\
\hline
\end{tabular}

Estadístico t en paréntesis; *Significativo al 10\%; **5\%; ***1\%.

De lo anterior, se puede evidenciar que las variables costo de ventas y valor agregado tienen significancia estadística, ya que la probabilidad del p>t es menor a 0.05. Adicional, se puede decir que cuando estas variables aumentan en un $1 \%$, las ventas aumentan en un $20 \%$ y $39 \%$ respectivamente. Esto se da, debido a que, en las ciudades, donde hay un mayor crecimiento empresarial, es donde adquieren productos y tecnologías más sofisticadas ( Fuenzalida, 2016). Adicional, se debe resaltar que cuando las empresas realizan inversión, como en tecnologías más sofisticadas para su producción, están permitiendo realizar procesos productivos más eficientes, para a su vez permitir una mayor adquisición de valor agregado en cada producto y, por tanto, tener más posibilidad de ser un determinante del desarrollo empresarial.

En los departamentos de ingresos menores se observa que para que las empresas se puedan sostener a lo largo del tiempo sobre otras, es necesario que puedan brindar un satisfactorio servicio y presenten una idea innovadora ante el mercado, para que de esta manera puedan ser competentes en el mercado regional. En otras palabras, así las 
empresas no sean lo suficientemente grandes, es necesario que generen un valor de agregado, no solo para que sean más competitivas, sino también porque es primordial para todo aquel que desea permanecer en el mercado, por ello, es un desafío para las empresas generar un valor agregado. (Ramirez Molina, Carval Herrera, \& Zambrano Meza, S.F).

En los departamentos de ingresos medios también es determinante el valor agregado y los costos de ventas, y cuando estos aumentan en un 1\% las ventas aumentan en un $78 \%$ y $11 \%$ respectivamente, el valor agregado por eso es relevante debido a la explicación de la tabla 4. Y en cuanto al costo de ventas, se relacionan con la publicidad ya que ayuda a conseguir nuevos clientes y a fortalecer la reputación de la empresa (good-will empresarial); Por tanto, se puede decir que la publicidad posee una finalidad extremadamente importante para cada empresa, debido a que ésta es un medio de información para conocer los productos y servicios. Pues, toda empresa, si desea ser reconocida en un mercado especifico, es necesario que, en sus costos, se encuentre un rubro de publicidad, ya que es ésta la que impulsa y estimula el crecimiento, a través del posicionamiento y el reconocimiento para la marca empresarial y los servicios que ellas ofrecen. Es por lo anterior, que toda empresa por pequeña o por grande que sea, busca de una u otra forma aumentar sus ventas y la visibilidad de su marca (Florez Jimenez, Florez Jimenez, Rodriguez Moreno, \& Vasquez Alamilla, 2014).

La información anterior se puede evidenciar en la regresión, debido a que logra tener choques positivos en el modelo, ya que por cada variación de un 1\% en la variable costos de ventas, el valor de las ventas varia en un $11 \%$, lo cual demuestra la gran relevancia de esta variable en los departamentos pertenecientes a niveles de ingresos medios según el PIB. Como se esperaba en los departamentos de ingresos más altos, los costos de ventas no son significativos, debido en primera instancia a que los costos fijos terminan siendo muy marginales al alcanzar economías de escala, además cualquier recurso adicional en los gastos de publicidad no genera tanto impacto como podría hacerlo antes de que la empresa consolidara un good-will (la variable publicidad, es uno de los componentes importantes de los costos de ventas).

Por otro lado, se identifica que la variable escolaridad es estadísticamente significativa para los departamentos con mayores ingresos, teniendo así una variación del 200\% por cada aumento en un $1 \%$ de los niveles de escolaridad. Ya que el aumento de un uno por ciento de capital humano, puede generar un impacto positivo debido a que nuevos conocimientos impulsan nuevas tecnologías, las cuales terminaran generando grandes cambios empresariales que potencian su productividad. Adicional en este departamento tipo 3 inciden fuertemente en esta variable, debido a la fuga de capital humano que se presentan en los departamentos con menores ingresos y que es recibida en los departamentos más grandes e industrializados. Por tal motivo el coeficiente de esta variable genera un impacto positivo en las ventas, provocando así, un incremento empresarial a largo plazo, lo que se ha sostenido desde periodos anteriores y que, de seguir las hegemonías empresariales, continuaran teniendo auges migratorios de manos de obra calificada. De igual forma, se nota que el valor agregado sigue siendo un gran determinante aun en los departamentos con ingresos superiores. Teniendo con esto una variación del 100\%, en ventas por cada variación del 1\% en esta explicativa. Cabe resaltar, 
que además de generar un valor adicional en el producto, genera una diferencia en el mercado, lo que produce un fuerte aumento en las ventas de la empresa.

\section{DISCUSION}

La investigación desarrollada tiene como finalidad encontrar los determinantes del crecimiento de las empresas reflejado en la variable ventas, que está planteada en el marco teórico como un buen indicador para este fin. De manera que se estudió un amplio juego de variables basadas en la teoría económica y administrativa, tanto de orden micro como macroeconómica, entre las que tuvieron una mayor capacidad explicativa y una mayor significancia estadística fueron costo de ventas, valor agregado y años de escolaridad. Por lo tanto, para lograr un análisis estratificado, se dividieron los departamentos por su PIB manufacturero en una división simple de 3, teniendo así a un $33,3 \%$ de los departamentos en cada una de las estratificaciones, lo que arrojó como resultado departamentos de ingreso 1 (bajo), 2 (medio) y 3 (alto), permitiendo un análisis más certero a la hora de dar recomendaciones.

Se escogió la variable costo de ventas esperando que la publicidad implícita en éste desprendiera un resultado positivo. No obstante, al correr los modelos de panel con efectos fijos, se encontró que los departamentos de tipo 1 y 2 reciben gran influencia de esta variable, esto debido a que estas empresas no requieren una estrategia de publicidad y marketing agresiva dada su naturaleza en el mercado competitivo, a diferencia de los departamentos tipo 3. Esto podría deberse a las ramas de actividades a las que se dedican, por ejemplo, las empresas que no necesitan ser publicitadas de forma fuerte en el país, debido a su estatus de monopolios naturales, por ende, presentan en menor medida inversiones en publicidad y ventas. Del mismo modo, los resultados evidencian que no existe una relación significativa entre las variables costos de producción, administración y ventas con el valor de las ventas en los departamentos tipo 3.

Según los resultados obtenidos, el comportamiento de las variables es diferente según el tipo de ingreso de los departamentos analizados. Ya que, para los departamentos de ingresos bajo y medio, las variables que inciden en el crecimiento empresarial son costo de ventas y valor agregado, sin embargo, los departamentos de ingresos altos, la variable que impulsa el crecimiento empresarial es el nivel de formación del personal y valor agregado. Lo cual explica la marcada diferencia que existe entre el crecimiento y desarrollo económico de cada región. Ya que, el capital humano ha sido ampliamente reconocido como una de las inversiones más rentables tanto individual (pues aumenta los ingresos y la calidad de vida), como socialmente, gracias a las externalidades positivas que genera. (Cepeda, L. s.f.). Adicional, como ya se había mencionado anteriormente, esto se conoce como, fuga interregional de cerebros, lo cual consiste en la migración de personas con un alto nivel de formación a regiones más prosperas, motivadas por una mejor calidad de vida y mejores condiciones laborales. Por tanto, regiones para las cuales el nivel de formación del personal es una inversión fundamental, y atraen mano de obra calificada y retienen la propia, son exactamente las mismas regiones que reportan un mayor crecimiento y desarrollo empresarial, reflejado en el índice de competitividad empresarial, Consejo Privado de Competitividad y Universidad del Rosario (2019). y en su PIB manufacturero. 
En contraste con otros estudios, el realizado por Trujillo y Iglesias (2012), destacan que los avances en los países desarrollados con respecto a la tecnología se complementan con el nivel educativo con el fin de aumentar la productividad de la empresa, Sin embargo, Acemoglu y Zilibotti (2001) recalcan que los países en vía de desarrollo pueden no incrementar su productividad en razón a que la baja calidad de la capacitación laboral dificulta la adopción de nuevas tecnologías. lo cual se puede aplicar a los departamentos tipo 1 y 2. El valor agregado fue un determinante indispensable en todos los departamentos estudiados, ya que es esta la variable que determina el tamaño de los flujos financieros al final del estado de resultados. Por su parte, la variable años de formación del personal es una de las variables que más dejó datos para el análisis a causa de que es fuertemente solicitada en las grandes capitales (departamentos de tipo 3). Esto también es explicado por la especificidad de los sectores que se desarrollan en estos departamentos, principalmente aquellos que necesitan mano de obra altamente calificada, por ejemplo, las empresas de tecnología.

\section{CONCLUSIONES}

En conclusión, el crecimiento empresarial, en el caso del sector manufacturero, está relacionado en gran parte con el departamento en que se encuentre, ya que según actividades priorizadas o las fortalezas del sector se permite una dinámica empresarial. Toda vez que, dependiendo de dichos factores, influirán más unas variables que otras; como es el caso de la educación, (medida en años de escolaridad), puesto que, en departamentos con mejores ingresos, se evidencia un impacto positivo en esta variable; caso contrario ocurre con los departamentos con menores ingresos. Por consiguiente, es fundamental tener en cuenta la referenciación de los departamentos, ya que de esta manera se permite alcanzar este tipo de diferenciación, lo que se traduce en una toma óptima de decisiones que involucren la contratación del personal que necesita una manufactura determinada.

Además de esto, el valor agregado es un factor, que independientemente del lugar en que se encuentre ubicada la empresa, va a traer consigo unas mayores ventas. Lo cual puede explicar por qué Colombia no es competitiva a nivel industrial, debido a la carencia de valor agregado en su oferta productiva de bienes y servicios. Una de las principales limitaciones que tuvo el estudio fue la escasa descripción de las variables que emite el DANE en la encuesta de estudio. Debido a esto, el análisis propuesto no pudo ser más profundo, sin embargo, la información disponible permite realizar un trabajo pionero en el que se vinculan la teoría microeconómica y administrativa y la cual permite dar luces del comportamiento del sector manufacturero en Colombia. Con base en el trabajo realizado se vislumbra una nueva línea de investigación relacionada con la medición de la productividad del sector, aspectos geográficos y espaciales.

Se espera que para futuras investigaciones se logre una mayor indagación en la forma en la cual fueron estructuradas las variables y adicionalmente, poder profundizar en los estudios de economía regional, buscando así encontrar las relaciones regionales que se encuentran en cada una de las perturbaciones que tienen las variables. Además, se puede evidenciar a lo largo del tiempo que sigue existiendo rezagos en la industria en los departamentos medios y bajos, que a su vez estos departamentos estos acompañados de indicadores socioeconómicos deficientes, lo cual puede explicar en cierta medida el poco desarrollo industrial. 
Finalmente, el presente artículo, permite realizar una recomendación para impulsar el crecimiento empresarial a nivel departamental, la cual está enfocada en generar políticas públicas que permitan retener la mano de obra calificada y a su vez atraer capital humano de otros departamentos del país, con el fin de que haya una mayor dinámica empresarial y crecimiento económico en la región. Ya sea en aumento de salarios, en condonar y/o disminuir deudas de estudios universitarios (ICETEX), beneficios a empresas que contraten jóvenes recién egresados y fortalecimiento de universidades e instituciones que generen capital humano de alta calidad.

\section{REFERENCIAS}

Aguilera, A. (2010). Direccionamiento estratégico y crecimiento empresarial: algunas reflexiones en torno a su relación. Pensamiento y Gestión, 85-106.

Andrade, J., Olivares, A., \& Robles, M. (2014). La planeación y control del costo de producción en las pequeñas empresas manufactureras, como herramientas que faciliten el cumplir tiempos de entrega del producto terminado. Epistemus, 75. Recuperado de: http://www.epistemus.uson.mx/revistas/articulos/17-11EMPRESAS MANUFACTURERAS.pdf

Banco de la República (2008). Determinantes del crecimiento de las empresas no financieras en Colombia: Ley de Gibrat y otras teorías. Bogotá, Colombia

Banco de la Republica. (2007). Bases para reducir las disparidades Regionales en Colombia. Cartagena. Documentos de Trabajo sobre Economía Regional (DTSER). Obtenido de http://www.banrep.gov.co/sites/default/files/publicaciones/archivos/DTSER-84.pdf

Blázques Santana, F., Dorta Velazquez, J. A., y Verona Marte, M. C. (2006). Factores del crecimiento empresarial.Especial referencia a las pequeñas y medianas empresas. Innovar, 44.

Blázquez, F. D. (2006). Concepto, perspectivas y medida del crecimiento empresarial. Cuadernos de Administración, 19(31), 165-195.

Blázquez, F. D. (2006). Factores del crecimiento empresarial. Especial referencia a las pequeñas y medianas empresas. Innovar, 16(28), 43-56.

Cañibano, C. (2011). El capital humano: factor de innovación, competitividad y crecimiento. Universidad Rey Juan Carlos. Recuperado de: https://www. navarra.es/NR/rdonlyres/D696EFD26AAA-4EF1-B414-E3A27109EA67/79785/14carolinacaibano.pdf

Cepeda Emiliani, L. (s.f.). ¿Fuga interregional de cerebros? El caso colombiano. Cartagena.

Consejo Económico y Social de Aragón. (2018). Mapa de crecimiento y rentabilidad empresarial en Aragón durante la década 2005-2015. Zaragoza, España.

Consejo Privado de Competitividad y Universidad del Rosario. (2019). Índice Departamental se Competitividad. Bogotá.

Cuervo, L. M. (2003). Evolución reciente de las disparidades Económicas territoriales en América Latina: estado del arte, recomendaciones de política y perspectivas de investigación. Santiago de Chile: Naciones Unidas. Obtenido de https://repositorio.cepal.org/bitstream/handle/11362/7296/1/50311842_es.pdf

DANE. (2016). Encuesta Anual Manufacturera. Bogotá.

Daza, J. (2015). La ley de Gibrat en las economías emergentes: El caso de Brasil. Departamento de Economía financiera y Contabilidad. Recuperado de: http://dehesa.unex.es/bitstream/handle/10662/3986/TDUEX 2015 Daza Izquierdo.pdf?sequenc $\underline{e=1 \& \text { isAllowed }=y}$

Flórez Jiménez, R., Flórez Jiménez, I., Rodríguez Moreno, R., y Vásquez Alamilla, M. (2014). La Publicidad, Una estrategia de éxito para las MyPimes de la Región Centro del sur Oeste del estado de Hidalgo. Inquietud Empresarial, 14.

Fuentes López, H. J., Jiménez Reyes, L. C., y Pérez Forero, N. A. (2019). La demografía industrial en Colombia: localización y relocalización de la actividad manufacturera. Cuadernos de Geografía: Revista Colombiana de Geografía, 28(1), 43-65.

Fuenzalida, J. (2016). Valor Agregado, Crecimiento y Comercio Internacional. Pharos. Gibrat, R. (1931). Les inegalite's economiques. Paris, France: libraire du Recaul Sirrey.

Grupo Bancolombia (2018). Grupo Bancolombia. Recuperado de https://www.grupobancolombia.com/wps/portal/negocios-pymes/actualizate/legal-ytributario/todo-sobre-las-pymes-en-colombia 
Guisado González, M., Coca Pérez, J. L., y Guisado Tato, M. (2016). Análisis de la relación tamaño empresarial-exportación desde un enfoque de complementariedad. Ekonomiaz, 57-75.

ICER (2016). Informe de coyuntura Económica Regional. Bogotá.

London, S., \& Formichella, M. (2006). El concepto de desarrollo de Sen y su vinculación con la Educación. Economía y Sociedad, 17-32.

Machado, H. P. (2016). Growth of small businesses: a literature review and perspectives of studies. Gestão \& Produção, 23(2), 419-432.

Moreno, J. L., y Ramos, J. L. (2013). Factores determinantes de la creación de empresas de base tecnológica en Colombia. Revista de Economía del Caribe, (12).

OCDE (2014). Perspectivas Económicas para América Latina 2016: Educación, Competencia e Innovación para el Desarrollo. París.

OCDE (2016). Impulsando la Productividad el Crecimiento Inclusivo en Latinoamérica. París: Publishing. Obtenido de http://oecd.org/latinamerica/Impulsando Productividad Crecimiento Inclusivo.

Paelinck, J. M. (2015). Modelos para datos espaciales con estructura transversal o de panel. una revisión. Estudios de Economía Aplicada, 23(1), 7-30.

Pérez, D., \& Castillo, J. (2016). Capital humano, teorías y métodos: importancia de la variable salud. Economía, Sociedad y Territorio, 16(52), 651-673.

Penrose, E. (1959). The Theory of the Growth of the Firm. Oxford: 3rd Edition Oxford University Press.

Perroux. (1963). Les industries Matrices et la Croissance d'une économie Nationale. Economie Appliqué, 16, 151-196.

Ramirez Molina, C., Carval Herrera, A., y Zambrano Meza, A. (S.F). La Creación de Valor agregado en las Empresas: Valor Económico Agregado, Eva y el Valor Agregado de Mercado. Saber, Ciencia y Libertad.

Rocca, E., García, D., \& Duréndez, A. (2016). Factores determinantes del éxito competitivo en la mipyme: un estudio empírico en empresas peruanas. Contabilidad y Negocios, 11(22), 52-68.

Romero de García, E. (2002). Claves para entender el desarrollo endógeno en la globalización. Ciencias Humanas y Sociales, 142-145.

Rodríguez, E. (2014). Los cuadrantes del desempeño regional en Colombia (2000-2012). Apuntes del Cenes, 33(57): 223-256.

Trujillo, J. C., y Iglesias, W. (2012). Determinantes del crecimiento de las micro, pequeñas y medianas empresas colombianas: el caso del sector metalmecánico. Semestre Económico, 15(32), 41-76.

Vázquez Barquero, A. (2018). Reflexiones teóricas sobre la relación entre el desarrollo endógeno y economía social. Revista Iberoamericana de Economía Solidaria e Innovación Socioecológica, 7, $11-22$.

Vergara, J. M. (2016). La obra de Amartya Sen. Iberian Journal of the History of Economic Thought, 3(2), 201-220.

Zuleta, J. M. (2007). Disparidades económicas regionales: un análisis de la distribución espacial de la población y del desempeño económico en Colombia. Observatorio de la Economía Latinoamericana.

Zuleta, J. M., López, L., \& Sánchez, D. (2008). Evolución de las disparidades económicas espaciales en Colombia: un análisis Regional. Medellín. 